\title{
Fathers and mothers developing skills in managing children's long-term medical conditions: how do their qualitative accounts compare?
}

\author{
V. Swallow, ${ }^{\star}$ H. Lambert, $\uparrow$ S. Santacroce $\$$ and A. Macfadyen ${ }^{\star}$ \\ ${ }^{*}$ School of Nursing, Midwifery and Social Work, University of Manchester, Manchester \\ $\dagger$ Department of Paediatric Nephrology, Newcastle upon Tyne Hospitals NHS Foundation Trust \\ $\ddagger$ School of Health, Community and Education Studies, Northumbria University, Newcastle upon Tyne, UK, and \\ $\S$ School of Nursing, University of North Carolina at Chapel Hill, Chapel Hill, NC, USA \\ Accepted for publication 16 December 2010
}

\section{Keywords}

chronic (health) conditions, development, fathers, mothers, skills

Correspondence: Veronica Swallow, School of Nursing, Midwifery and Social Work, University of Manchester, Room 4.315, University Place, Oxford Road, Manchester M13 9PL, UK

E-mail:veronica.swallow@ manchester.ac.uk

\begin{abstract}
Background Little is known about the respective experience of fathers and mothers within couples when managing their child's long-term medical condition. This study therefore aimed to obtain and compare fathers' and mothers' accounts of managing long-term kidney conditions. Methods Qualitative study involving individual then joint semi-structured interviews with 14 couples (biological fathers and mothers of 15 children whose care is managed at a specialist unit). Interviews were digitally recorded, transcribed and analysed using Framework Analysis. Transcripts within and across couples were compared.

Results Fathers and mothers made a significant contribution to management and a key theme identified was 'developing skills' in: information processing, sharing/negotiating caregiving, restraining children, adapting to treatment regimens and communicating. Although skill development was often a challenging and uncertain process, both fathers and mothers wanted to and did participate in caregiving; they often negotiated this with each other to accommodate caring for other children, paid employment and to provide mutual practical and emotional support. Developing skills in holding their child for procedures and treatments was a major concern, but it was fathers who assumed the 'protector' role and worried more about their child's long-term health and well-being, while mothers concerned themselves more with current clinical issues and maintaining relationships with professionals. Expressing appreciation for fathers' and mothers' skill development may promote good 'working relationships' between professionals and both parents over the many years of the trajectory.

Conclusion Developing skills for home-based caregiving of long-term conditions is a challenging and uncertain process. Both parents often participate in caregiving, and the findings reported here may help professionals decide how best to support both parents in their home-based caregiving.
\end{abstract}

\section{Introduction}

Parents' contributions to condition management are well documented but until recently research tended to focus on mothers as main respondents (Eiser \& Havermans 1992; Gallo \& Knafl 1998; Knafl \& Zoeller 2000; Swallow \& Jacoby 2001; Sullivan-Bolyai et al. 2006; Aldridge 2008). This may be because mothers are more often present during clinical consultations, therefore more likely to be approached about research and their accounts used as a proxy for fathers' accounts (Swallow 2008). However, most children live with two biological parents (Census-Bureau 2004; ONS 2009) and fathers' contributions to family life are 
increasingly acknowledged by society; this includes their contributions to management of medical conditions (Burgess 2005; Gavin \& Wysocki 2006; Pelchat et al. 2007; Swallow 2008; Swallow et al. 2011). Poor compliance with therapy, for whatever reason, may be associated with increased morbidity and mortality, while good parental understanding may enhance parents' active participation (Eiser et al. 1992; Soliday et al. 2001; DoH 2004, 2006; Gavin \& Wysocki 2006; Miller \& MacDonald 2006; Swallow et al. 2008); moreover, research highlights the importance of '...giving voice to the previous silence of fathers with chronically ill children' (Peck \& Lillibridge 2005, p. 43). Nevertheless, fathers' views and the parental partnership are still underrepresented in the literature (Ramchandani \& McConachie 2005), meaning that fathers' contributions to caregiving within couples remain poorly understood by health professionals (Gallo \& Knafl 1998; Knafl \& Zoeller 2000; Pelchat et al. 2003; Swallow 2008; Hill et al. 2009). During recent research exploring family learning in long-term condition management, fathers and mothers sometimes reported discrepant views during joint interviews (Swallow 2008). Further research was recommended involving individual and joint interviews with mothers and fathers to inform practice and policy. Therefore, further study of the nature and significance of parents' respective views was undertaken (Swallow et al. 2009); 'developing skills' was a key emergent theme, and this paper presents a detailed report of this aspect of the study.

\section{Methods}

\section{Aims \& objectives}

- To determine mothers' and fathers' individual and joint views of their own and their partner's roles and responsibilities.
- To compare individual and joint accounts.

\section{Design}

To obtain in-depth understanding of mother-father dyads, we adopted an interpretative approach using qualitative methods; this approach stresses the importance of interpretation as well as observation in understanding the social world so was appropriate to address our aims (Ritchie \& Lewis 2003; Green \& Thorogood 2004). As little consensus exists about the use of individual or joint research interviews with couples (Thomas 1987; Chesla 1995; Racher et al. 2000), we obtained both individual and joint accounts.

\section{Sampling}

Using kidney conditions as an exemplar, parents who had managed their child's condition for at least 12 months before study recruitment were selected from a children's kidney unit database in the North of England. To maximize the diversity of our sample, we used purposive sampling (Ritchie \& Lewis 2003) based on the child's age, gender and level of management intervention needed (Table 1). Married/unmarried couples and step/ adoptive parents were invited.

Fifty-nine couples received postal invitations to participate. The resulting sample involved 14 couples (biological, Caucasian parents of 15 children) representing a range of socioeducational backgrounds (Table 2).

All parents provided signed consent after receiving assurances about anonymity and confidentiality; all had previously performed at least six management skills related to their child's condition (Table 3, Column 1).

Table 1. Sampling framework

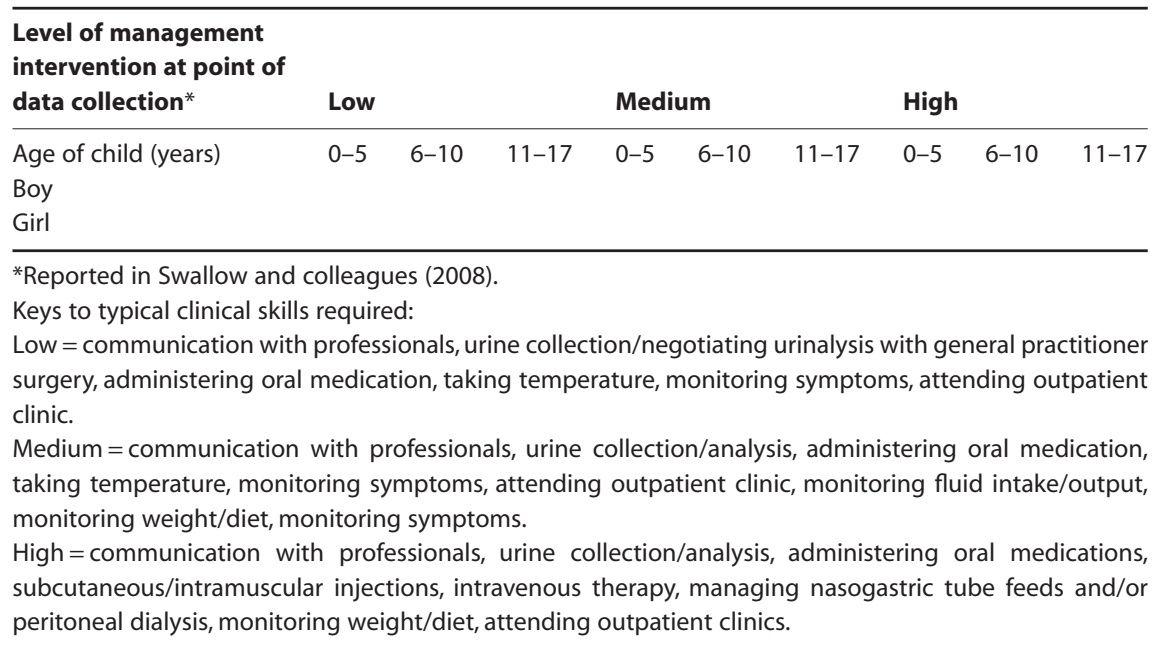




\begin{tabular}{llll}
\hline Dyad number & $\begin{array}{l}\text { Parents' educational } \\
\text { achievement since } \\
\text { leaving school* } \\
\text { Mother/father }^{*}\end{array}$ & $\begin{array}{l}\text { Household post-code } \\
\text { socio-economic rating } \\
\text { A-E† }\end{array}$ & $\begin{array}{l}\text { Level of intervention } \\
\text { at study recruitment }\end{array}$ \\
\hline 1 & NVQ3/RGN & $\mathrm{CD}$ & Low \\
2 & Degree/Degree & $\mathrm{ABC}$ & Low \\
3 & Degree/RGN & $\mathrm{AB}$ & High \\
4 & Degree/Degree & $\mathrm{ABC}$ & Medium \\
5 & NVQ/0 & $\mathrm{CDE}$ & Medium \\
6 & Degree/Degree & $\mathrm{ABC}$ & Medium \\
7 & GNVQ/Degree & $\mathrm{CD}$ & Low \\
8 & Certificate/Certificate & $\mathrm{ABC}$ & Medium \\
9 & NVQ/Diploma & $\mathrm{CD}$ & Low \\
10 & Degree/Degree & $\mathrm{ABC}$ & Medium \\
11 & Degree/Degree & $\mathrm{ABC}$ & Low \\
12 & Diploma/0 & $\mathrm{BC}$ & Low \\
13 & Degree/Degree & $\mathrm{CD}$ & Low \\
14 & O/0 & $\mathrm{BC}$ & Medium \\
\hline
\end{tabular}

Source: http://www.businessballs.com/demographicsclassifications.htm

*Keys: NVQ, National Vocational Qualification, is at certificate level, normally related to a particular skill (e.g. bricklaying, childcare); RGN, registered general nurse; GNVQ, General National Vocational Qualification.

tKeys: A - higher managerial, administrative or professional; B - intermediate managerial, administrative or professional; C - supervisory or clerical, junior managerial, administrative or professional/skilled working class, skilled manual workers; D - semi-skilled and unskilled manual workers; E - casual or lowest-grade workers.
Table 2. Sample characteristics of participating parents

Table 3. Example of home-based clinically focused skills performed by mothers and fathers

\begin{tabular}{|c|c|}
\hline Examples of skills parents performed at start of current study & Examples of additional skills reported in current study \\
\hline $\begin{array}{l}\text { Understand investigations } \\
\text { Collect and/or test urine } \\
\text { Administer (sometimes complex and often changing) medications } \\
\text { Administer gastrostomy or nasogastric tube feeds } \\
\text { Insert nasogastric tube } \\
\text { Monitor and record blood pressure } \\
\text { Manage central line care } \\
\text { Monitor diet and fluids } \\
\text { Recognize importance of subtle but significant clinical changes } \\
\text { Communicate clinical changes or new signs and symptoms effectively } \\
\text { to clinical staff } \\
\text { Coordinate the many aspects of home-based care } \\
\text { Support child's management while also promoting their development } \\
\text { Maintain daily family life and own work commitments }\end{array}$ & $\begin{array}{l}\text { Communicate effectively with members of the multidisciplinary team about } \\
\text { treatment regimens } \\
\text { Be hyper-vigilant, monitoring and reflecting on what professionals say and do } \\
\text { Disengage when child was an inpatient to allow time for personal reflection on } \\
\text { the situation } \\
\text { Process large amounts of clinical/systems information } \\
\text { Develop/maintain good relationships with professionals } \\
\text { Share first-hand information from professionals with partner to inform shared } \\
\text { decision making } \\
\text { Make decisions based on second-hand information } \\
\text { Apply urine bags/pads to collect urine samples } \\
\text { Restrain child for investigations and to administer treatment regimens } \\
\text { Ensure medications taken as prescribed } \\
\text { Keep child calm } \\
\text { Live with sustained uncertainty } \\
\text { Communicate with the child about the condition according to their ability to } \\
\text { understand } \\
\text { Adapt to treatment side effects on the child } \\
\text { Keep self-calm } \\
\text { Ask clinical questions of multidisciplinary team members } \\
\text { Adapt family life, the home environment and work commitments to encompass } \\
\text { child's clinical needs } \\
\text { Share and negotiate caregiving with partner }\end{array}$ \\
\hline
\end{tabular}

\section{Data collection}

Twenty-eight individual, then 14 joint semi-structured interviews (lasting on average $30 \mathrm{~min}$ each) were conducted by A. M. in family homes or parent's workplaces, supported by a topic guide (Table 4), digitally recorded and later transcribed verbatim. As data management proceeded, emerging themes supplemented interview topics. 
Table 4. Summary of interview topics (T) and examples of questions/openings used (Q)

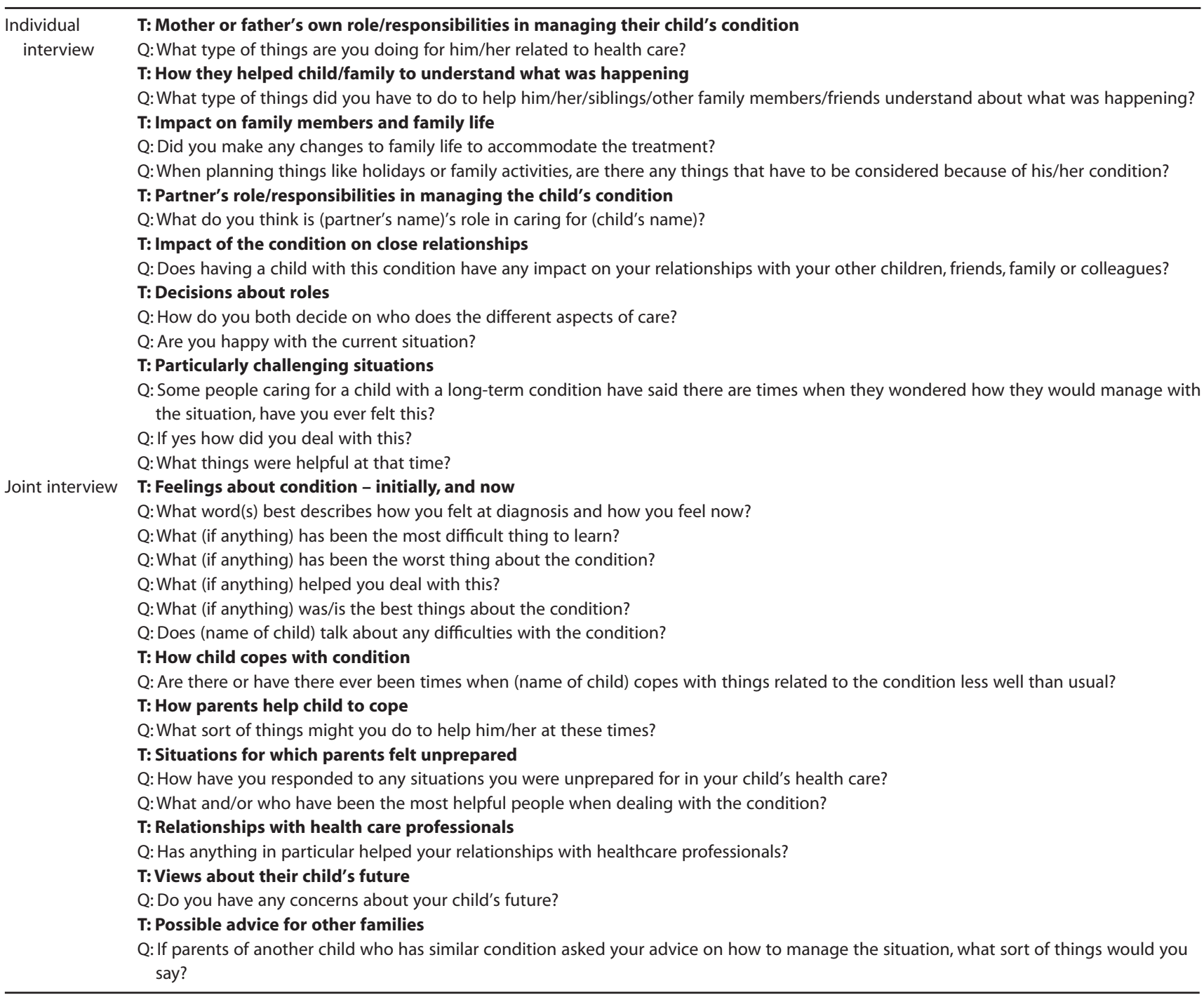

\section{Data analysis}

Data were analysed using Framework. Data management was supported by Microsoft Excel (Ritchie \& Spencer 1994; Ritchie \& Lewis 2003; Swallow et al. 2003). Framework borrows its principles and approach to implementing these principles, from different epistemological traditions within the social science field; it is this eclecticism that has remained its strength throughout its development as an analytical process (Ritchie \& Spencer 1994; Ritchie \& Lewis 2003). Framework's ontological position adheres most closely to subtle realism (Hammersley 1992), which accepts that the social world does exist independently of individual subjective understanding, but that it is only accessible in qualitative research via partici- pants' interpretations, which may then be further interpreted by the researcher. Framework is systematic, thorough and grounded in the data but also flexible and enables easy retrieval of data to show others; it has an overt policy orientation with an end point of developing strategies for practice on the basis of analyses (Ritchie \& Lewis 2003; Green \& Thorogood 2004). These combined factors meant Framework was the appropriate method for the current study which builds on emergent findings from an earlier study that used a grounded theory approach.

In the current study, the iterative process of data management involved two authors (A. M. and V. S.) moving backwards and forwards between the stages of Framework using the most recent iteration of the process described by Ritchie and Lewis 


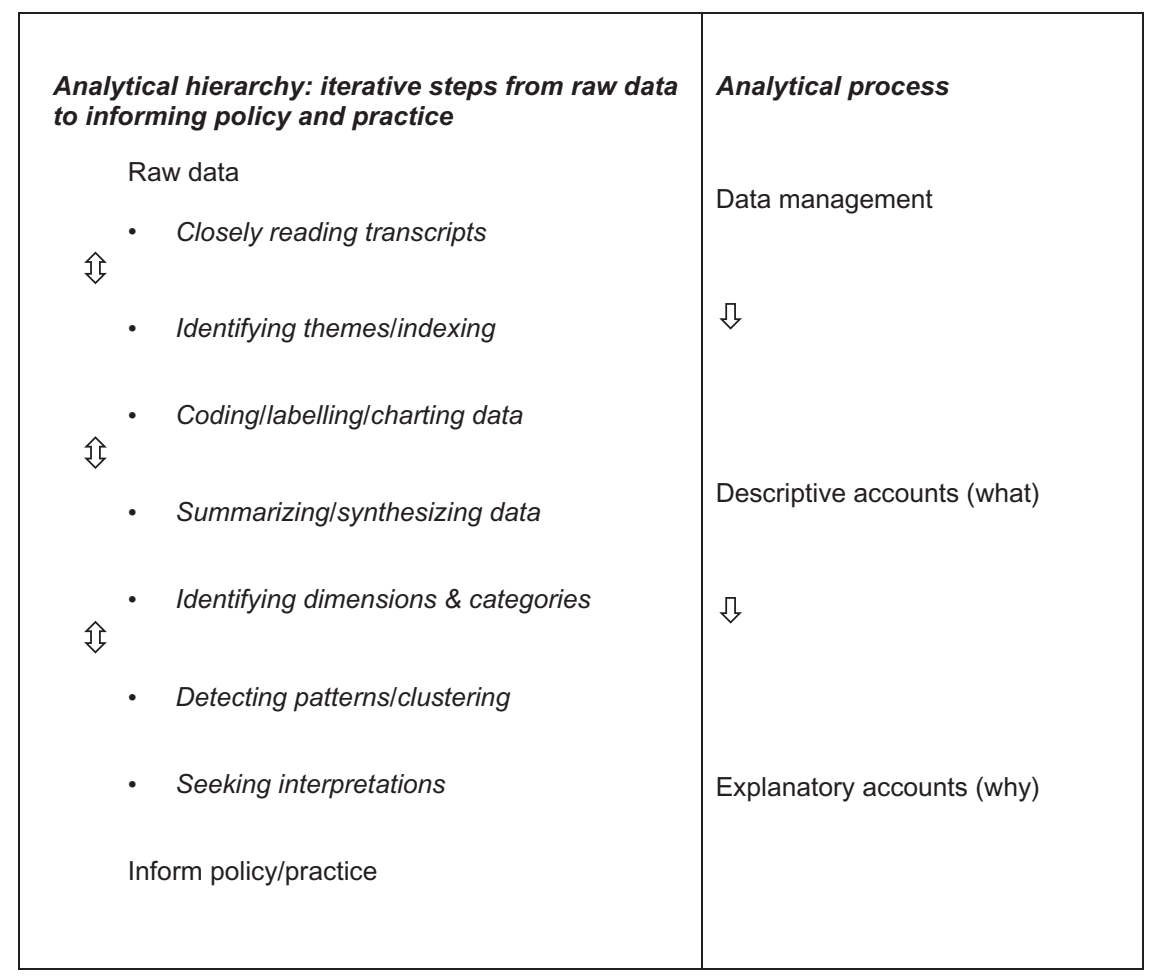

Figure 1. Analytical hierarchy and processes of framework analysis, adapted from Ritchie and Lewis (2003).
(2003) as summarized in Fig. 1, and supported by Microsoft Excel (Swallow et al. 2003). Thus, after closely reading all transcripts, a coding framework of themes and sub-themes was developed and then applied (manually) to all transcripts; data summaries and key direct quotations were labelled using the numerical reference to the appropriate theme/sub-theme and then 'lifted' to a Microsoft Excel spreadsheet for charting and referenced back to the page number on the original transcript for later retrieval when reporting. The process of charting within the theme 'developing skills' is illustrated in Table 5.

This enabled comparison within and between cases (which was also facilitated by colour coding mother and father data sets) and rearrangement of the data case-by-case and by thematic content for subsequent descriptive analysis and abstraction.

Working through the raw data with this level of intensity helped us identify the lines of enquiry to pursue during descriptive analysis, the main purpose being to unpack the content and nature of each theme and to display the data in a way that makes distinctions that are meaningful and provides content that is illuminating. At the first stage of abstraction (Table 6, Column 2 ), descriptions stayed close to the original data so that the initial elements can be seen within the audit trail.

In the more abstract categorizations (Column 3), three different classifications began to emerge: the first concerns fathers' and mothers' mutual emotional support during management (described and discussed in more detail elsewhere); the second, the physical support they provided for their child and each other; and the third, communication that related to these (these last two underpin the results presented here). By retaining the connection between original data and the categorizations in this way, the elements that were aggregated remained available for retrieval later in the analysis if needed (Ritchie \& Lewis 2003; Green \& Thorogood 2004). During this process the substantive content and elements of the theme 'developing skills' were identified by looking across all cases in the study and noting the range of perceptions, views, behaviours and experiences that had been labelled as part of the theme and it became clear, for instance, that mothers and fathers repeatedly referred back to their concerns about their child at an earlier point in the trajectory and how they had since developed the ability to deal with situations by becoming better skilled at processing information, sharing and negotiating caregiving, restraining their child, adapting to treatment regimens and communicating about the condition with their child, professionals, family and friends. In addition, it was noted that it was consistently fathers who took responsibility for 'protecting' their child during upsetting events.

All authors then worked independently with data samples, searching for patterns within the data, mapping connections and seeking explanations for patterns before comparing and 
Table 5. Example of charting process used within the theme 'developing skills' for one respondent

\begin{tabular}{|c|c|c|c|c|c|}
\hline \multicolumn{6}{|c|}{ Theme: developing skills } \\
\hline & $\begin{array}{l}\text { Sharing/negotiating } \\
\text { caregiving }\end{array}$ & $\begin{array}{l}\text { Information processing/ } \\
\text { dealing with large } \\
\text { amounts of information }\end{array}$ & Restraining children & $\begin{array}{l}\text { Adapting to treatment } \\
\text { regimens }\end{array}$ & Communicating \\
\hline \multirow[t]{3}{*}{ Respondent F2 } & $\begin{array}{l}\text { Medicines - nightmare at } \\
\text { first! Now worked out } \\
\text { how to manage better } \\
\text { (p. 13) }\end{array}$ & $\begin{array}{l}\text { Information about initial } \\
\text { tests - timing (pp. } \\
11-12 \text { ) }\end{array}$ & $\begin{array}{l}\text { Was difficult when they } \\
\text { were younger but now } \\
\text { cooperates better } \\
\text { (parents learned to } \\
\text { always tell them what } \\
\text { was going to happen } \\
\text { first) (p. 9) }\end{array}$ & $\begin{array}{l}\text { Explanations about initial } \\
\text { tests was hard to } \\
\text { understand at first but } \\
\text { got easier (good } \\
\text { quotation) (p. 11) }\end{array}$ & With grandparents (p. 13) \\
\hline & $\begin{array}{l}\text { Urine collection - (found } \\
\text { best way was one } \\
\text { holding child other } \\
\text { managing equipment) } \\
\text { (p.16) }\end{array}$ & $\begin{array}{l}\text { Trying to get it straight in } \\
\text { your mind (p. 15) }\end{array}$ & & & $\begin{array}{l}\text { Learning to ask them } \\
\text { (professionals) } \\
\text { questions the best way } \\
\text { (p. 11) }\end{array}$ \\
\hline & & $\begin{array}{l}\text { Good to have someone } \\
\text { put it into context for } \\
\text { you as early as possible } \\
\text { (p. 17) }\end{array}$ & & & $\begin{array}{l}\text { Making sure the child is } \\
\text { up to date with } \\
\text { progress and } \\
\text { treatments (p. 4) }\end{array}$ \\
\hline
\end{tabular}

Table 6. Example of the way Framework was used for descriptive and associative analysis in the theme 'developing skills'

\begin{tabular}{|c|c|c|c|c|}
\hline \multicolumn{5}{|c|}{ Theme: developing skills } \\
\hline Column 1 & Column 2 & Column 3 & Column 4 & Column 5 \\
\hline Respondent & Data summary & Dimensions identified & Categories & Clusters \\
\hline M\&F1 - joint & $\begin{array}{l}\text { Father realized if he took over dialysis when Mum was } \\
\text { upset she coped better afterwards. } \\
\text { Mother could ask him to do anything. } \\
\text { Father drove long distances daily to be with them in } \\
\text { hospital. }\end{array}$ & $\begin{array}{l}\text { Took over care } \\
\text { Accommodating/willing } \\
\text { Being with them } \\
\text { Protectiveness } \\
\text { Taking over if necessary }\end{array}$ & $\begin{array}{l}\text { Physical support } \\
\text { Physical support } \\
\text { Emotional support } \\
\text { Emotional support } \\
\text { Physical support }\end{array}$ & $\begin{array}{l}\text { Physical support } \\
\text { Taking over care } \\
\text { Practical support } \\
\text { Emotional support } \\
\text { Dad being with them } \\
\text { Being strong }\end{array}$ \\
\hline M\&F5 - joint & $\begin{array}{l}\text { Reassuring one another } \\
\text { Keep calm and stop mum from 'going to pieces' (father) }\end{array}$ & $\begin{array}{l}\text { Reassurance } \\
\text { Being strong }\end{array}$ & $\begin{array}{l}\text { Communication } \\
\text { Emotional support }\end{array}$ & $\begin{array}{l}\text { Communication } \\
\text { Reassurance }\end{array}$ \\
\hline
\end{tabular}

Keys to column headings:

Data summary: Summarizing the content of data excerpts to best retain the context and essence of the point and without losing the language or voice of the respondent.

Dimension: The substantive content and dimensions are identified; descriptions stay close to the original data so that initial elements of the data can be seen. Category: The analyst (1) begins to assign 'labels' to the data that have moved beyond the original text and begun to interpret the data in a more conceptual way; (2) the categorizations used show that the same features are appearing in different cases; and (3) other categories are emerging that are very similar in conception and could be collectively described under a slightly broader heading.

Cluster: Patterns of association or linkages within the theme and across the data set.

discussing these collectively until achieving a consensus. Constant comparison between transcripts (within and across couples) opened up meaning in the text until no new themes emerged, by which time we had undertaken 28 individual and 14 joint interviews and recruitment ceased. Overall anonymized findings were fed back to parents. All parents received postinterview thank-you letters and invitations to discuss any management concerns arising during study participation with a member of the clinical team, one couple accepted and their case is being reported elsewhere.

\section{Ethical considerations and rigour}

Approval to conduct the study was obtained from a Department of Health local research ethics committee and the NHS Trust Research and Development Department. Participants were told that data would be anonymized and stored in a secure place accessible only to V.S. and A. M. (thus ensuring clinical members of the research team who were also responsible for children's management could not recognize the source of quotations from among their patients' parents), and that declining to enrol or 
later withdrawing would not jeopardize their child's subsequent care or relationships with professionals. Every effort was made to ensure consent was informed and freely given by encouraging parents to consider and/or discuss the project with relatives or primary-care staff before deciding whether to participate (UN 1989; McIntosh et al. 2000; Allmark 2002; Alderson 2005). Written and verbal information was provided and written consent obtained from both parents. Data were collected by researchers who were previously unknown to the parents, thereby reducing the possibility of participants providing socially desirable responses. To ensure trustworthiness and credibility, reduce potential bias and enhance theoretical sensitivity, we incorporated reflexivity into the data management process regularly considering whether analysis might have been compromised through premature closure in favour of our own preconceived ideas (Ritchie \& Lewis 2003; Green \& Thorogood 2004). Because the study involved only one site, information about diagnoses that could lead to easy identification of participants is omitted from the reporting. Independent data sample reviews were discussed by all authors until a consensus was reached; respondent codes were available only to the authors who collected data. An audit trail of data management processes was maintained to ensure transparency of the research procedure.

\section{Results}

Differences existed between maternal and paternal accounts within and across couples. This section juxtaposes data excerpts ( $\mathrm{F}=$ individual interview with father; $\mathrm{M}=$ individual interview with mother; $\mathrm{M} \& \mathrm{~F}=$ joint interview with mother/father) with discussion to illustrate individuals' accounts within the dimensions of the theme 'skill development'.

\section{Processing large amounts of information}

Developing skills in processing large amounts of information about the clinical problem, its consequences and treatment regimens as well as the processes of the healthcare system was a challenging and uncertain process. Fathers tended to prefer the 'bigger picture':

... I said [to professionals] I need as much information as you can throw at me, I'll say when it's too much, so they started telling me and I said 'Stop, that's enough'. [F6]

and generally took a long-term view:

It's [son's condition] a bomb ticking away in the background ... you wonder what he'll be like in 10, 15, 18 years. [F6]
Mothers, however, focused on the short term in relation to dealing with the information provided by professionals:

I still don't think long term ... he [husband] wonders more about what's going to happen later; I'm more here and now. [M6]

and coming to understand about the range of essential treatments the child may need and their possible impact:

My biggest fear is him [son] having a line [central venous] in as [for instance] he's so ridiculous about getting his blood pressure done and a needle in. [M9]

For mothers in particular, assimilating information from professionals about the importance of correctly managing their child's condition at home and that their own actions may result in negative outcomes for their child led to several concerns. For instance; one mother vividly described her reactions to administering home-based peritoneal dialysis (PD):

I used to sit there... hyperventilating ... feeling sick ... it was horrible ... trying to control that, learning not to panic ... don't think I ever actually mastered that [realization that the child may develop an infection and she may be responsible]. [M7]

In joint interviews, some parents described role divergence when dealing with large amounts of information provided by professionals; mothers tended to become emotional, whereas fathers tended to be more calm and fathers were often hypervigilant, monitoring and reflecting on what professionals said and did, while one mother noted that her husband was 'more of a thinker' while she 'takes what we are told at face value'. [M\&F10]

\section{Sharing/negotiating caregiving}

There was evidence of role divergence with fathers sometimes disengaging from the clinical context when their child was an inpatient to allow time for personal reflection about the situation, but at home the same fathers shared caregiving to support the mother. One mother explained:

... if I was freaking out... he'd [father] take over, he probably put her on PD more than me ... wiring her up to something I knew was going to cause a lot of pain was just unbelievable, so he'd put her on and I'd hide in the bathroom and cry. [M7]

Parents recalled how balancing the whole family's needs in order to accommodate the ongoing demands of the condition 
required careful planning and negotiation so, for instance, couples explained that if a clinical emergency arose at home and the child needed to attend hospital, they learned to 'call an ambulance rather than taking the whole family to hospital in the car' [F1] as they did initially; this meant one parent stayed at home with the other children, which was less disruptive for the family. If the father generally worked away from home the mother gave all home-based clinical care and attended hospital. Other parents shared care and negotiated tasks:

I do some care and appointments that fit in with my work commitments; we negotiate other tasks. [M14]

However, all participants liked to be included in decision making about clinical management, so when professionals discussed clinical issues, including treatment options, with one parent the information subsequently needed to be relayed by that parent to their partner so they could make a joint decision:

... but it's nice [for us both] to have information first hand rather than one of us getting it and passing it on, so then we can share the decision and work out between us what it might mean. [M7]

\section{Restraining children}

To ensure investigations and treatments were successfully completed as quickly as possible for the child's benefit, children often needed restraint; while this could be distressing for children and parents, mothers and fathers felt it was important for one of them to assist with holding their child. Restraint was referred to spontaneously by only two mothers:

... when first ill she was an absolute bloody nightmare! ... would take two or three of us to hold her down and get it [medicine] in, but she just takes it now, even if she doesn't like the taste. [M13]

Another described central line flushing:

When you're not trained in using needles/syringes [holding her still while holding and using a syringe and needle at the same time] is initially very difficult...

subsequently her child was discharged when both parents had gained sufficient experience, but anxiety prevailed:

We used to draw straws ... 'You do it' [mother to father], then 'No, you put it in [needle, attached to syringe to inject the child] and I'll hold him' [father to mother]. [M\&F6]
Several fathers spontaneously talked about 'holding' their child for procedures such as urine collection, venepuncture, blood pressure measurement, nasogastric tube insertion, applying local anaesthetic cream and giving medicines, all appearing to regard this as the father's personal responsibility, for example:

I try to keep him calm for bloods. [F6]

Similarly, when the issue of 'holding' was raised by one father in a joint interview, the mother attributed her husband with the ability to manage 'better' than she:

He can be more 'remote' when holding him for tests. [M\&F2]

\section{Adapting to treatment regimens}

Parents described how they learned to adapt to accommodate treatments and changes in the child caused by the condition or its treatment regimens, ensure medications were delivered effectively and live with sustained uncertainty. For example, some described strenuous efforts to adapt their home to accommodate essential clinical supplies/equipment: regular urine collections could involve '... piles of pads and things to put in his nappy' [M10], while PD meant installing a sink and storage in the bedroom:

You don't want a dialysis machine in your kid's bedroom; it's not nice, but obviously that became where the equipment was kept. [M13]

or the garden shed:

... the bags and things, I had a shed outside, it was full of them. [F11]

A prominent problem was supporting children to take unpleasant medicines:

There were problems with her taking meds [medicines] at first but it's better now; we have [learned to] make sure that she has grown up knowing she has to have medicines and that she knows that otherwise she will be ill, and that that [being ill] is not a good experience. [F1]

Success at ensuring the child took treatments was described by one father as 'probably the greatest achievement' [F10]. Concerns about side effects of steroid treatment featured highly with mothers and fathers and related to excessive weight gain leading to children being teased or difficulty obtaining wellfitting shoes and clothes: 
I had to go up a size to get the width of shoes; she was literally falling out of the sides of her shoes. [M3]

and behaviour changes:

His whole manner changes, can almost see the frustration in him and that's hard because you try to keep the boundaries with his behaviour. [F7]

Mothers and fathers highlighted the stress of learning to live with sustained emotional uncertainty:

Uncertainty was the worst thing to deal with. [F1]

and:

... a hard slog ... living with it [long-term condition] every day and you don't know if it is forever. [M7]

but fathers, in particular, saw adaptation as essential:

The sooner you get used to the idea that you are in it for the 'long haul', the sooner you can make all the changes and adapt. [F6]

\section{Communicating}

Learning to communicate appropriately and effectively with professionals was important to mothers and fathers. Mothers focused on balancing their child's welfare with maintaining good relationships with professionals:

... I said [to the doctor] 'If you can't get it [venepuncture] first time get someone else' ... I felt awful saying it but thought it better than when I was annoyed with them, and he's [son] being prodded and poked. [M7]

Fathers, on the other hand, developed proactive information seeking strategies when communicating with professionals, for instance:

You got to learn the questions to ask them to get the information you want. [F6]

and one father believed he regained 'some control in time by reviewing what you know and what will happen next'. [F1]

Communicating with each other was also a key issue, with mothers and fathers describing caregiving as 'team working'; this could result in a complementary approach as explained by one father who described how they each take '... different approaches to keeping on top of what is happening' [F4]. Alternatively, mothers described how a compensatory approach worked:
I can take stress more easily... he gets very stressed easily, I've learnt to be calmer. [M8]

Mothers tended to discuss active mutual support in practical terms, for example:

It was just the two of us, he was trying to squeeze the urine collection pad really tight, I was trying to do the syringe bit, we just couldn't get anything out! [M\&F2]

When talking about communicating with the child, parents tended to emphasize how they were sometimes more lenient with their child than they had been before the onset of the condition:

... significantly changed our approach to discipline with him. [F6]

and:

I [now] get sentimental about him at times. [F10]

One father explained that the illness experience altered the way he communicated with his son:

... got very close to him in ITU [Intensive Therapy Unit], made solid eye contact with him, reassuring him, whispering to him, playing games through bars of the cot. [F5]

During the interviews parents often recalled events from several years ago when the child was first diagnosed with the condition:

We comforted him [when young] and tried to make him comfy with cuddles and distractions. [F5]

Because professionals had discussed the condition and its treatments primarily with parents, mothers and fathers of older children learned to 'make sure he [son] has the same information as we do' [M1] so that the affected children did not feel excluded.

Mothers alluded to the challenge of communicating with relatives and friends about their child's condition:

... it doesn't just affect immediate family . . . but everyone around you ... very emotional, feeling of helplessness ... people ask 'when will he get better?' we just don't know. [M3]

Being available to attend hospital for follow-ups or unplanned admissions was stressful for both parents, but fathers in particular highlighted the importance of communicating with employers to ensure:

... no problems from [employer] if I have to go to hospital. [F6]

... school and work very supportive. [F9]

\section{Discussion}

The quality of parents' contributions to expert management of their child's medical condition may affect children's clinical 
outcomes. Poor compliance with therapy, for whatever reason, may be associated with increased morbidity and mortality, while good parental understanding may enhance parents' active participation (Eiser et al. 1992; Soliday et al. 2001; DoH 2004, 2006; Gavin \& Wysocki 2006; Miller \& MacDonald 2006; Swallow et al. 2008). Supporting both parents from early in the trajectory in their efforts to manage the condition may therefore be important in trying to reduce length or frequency of hospital admissions and improve outcomes (Eiser et al. 1992; Hovey 2006; Swallow 2008) over the many years of the trajectory.

Although limited data currently exist to inform practitioners about parents' respective caregiving contributions, previous work does indicate that parents often hold shared views of a condition's impact on family life (Eiser \& Havermans 1992; Costigan \& Cox 2001; McBride et al. 2005; Barakat et al. 2006). Our study builds on these findings by highlighting new insights into maternal and paternal experiences and suggests a more complex picture of skill development within caregiving than previously reported. The skills mothers and fathers reported are typical of those required in management of other long-term conditions; therefore, our findings may have wider implications in other child health settings.

For mothers and fathers, developing skills in holding their child for procedures and administering treatments was a major concern, but it was fathers who assumed 'responsibility' for keeping the child calm, protecting their interests and minimizing their stress. Others have shown that fathers can adopt the protector role in the Neonatal Intensive Care Unit, for instance (Arockiasamy et al. 2008); it appears from our data that the protector role is also adopted by fathers of children with long-term conditions. This suggests that professionals could openly discuss this 'protector role' with parents early in the trajectory. When processing information mothers and fathers both wanted to be involved, so professionals may seek to form alliances with fathers as well as mothers where both parents are involved in the child's care, giving information to both so that neither parent gets 'second-hand' information. Professionals may find it helpful to be aware of the possibility of fathers' hyper-vigilance early in the trajectory as reported in this study and referred to by other authors (Neil-Urban \& Jones 2002; Arockiasamy et al. 2008). Fathers worried more about their child's long-term future than mothers; worry about the future and the need for control is a key element in traditional constructions of masculinity and the male role as family provider/ protector (McGrath \& Chesler 2004). In line with other reports (Chesler \& Parry 2001; McGrath \& Chesler 2004), our findings suggest a need for professional interventions with fathers that help them develop coping strategies for dealing with worry and inform them how their involvement can promote their child's well-being.

Our study extends previous research that recommends intervening with fathers early in the trajectory (Clark \& Miles 1999). Time constraints may mean clinicians' information provision strategies are not specifically designed to meet both parents' needs, but our data suggest that if clinicians are concerned about communicating effectively with fathers as well as mothers, they may consider offering flexible hours for discussions to accommodate parents alternative commitments such as paid employment or caring for other children. Actively encouraging and expressing appreciation for fathers as well as mothers in their skill development and caregiving contributions may be important in promoting good 'working relationships' between professionals and both parents over the many years (sometimes decades) of the trajectory, and may help prevent misunderstandings, conflict and parental burnout.

\section{Study limitations}

Our findings should be considered in light of some limitations. Sampling involved only one unit; therefore, care needed to protect anonymity and confidentiality led to some limitations in reporting. For example, because a wide range of conditions are collectively referred to as long-term kidney conditions and some of these conditions are rare, we omitted from our reporting any person-identifiable information such as children's diagnoses, aspects of treatment or parents' occupations that might make them or their condition identifiable to the reader. Children were sampled according to level of management intervention at recruitment, but for some this had varied among low, moderate and high before study participation so parents' accounts may reflect this variation. All participants were Caucasian and from primarily intact families (so not necessarily representing the population base in all units), and data collection involved a 'snapshot' of one time point. When no new themes emerged and theoretical saturation was reached, 59 couples had been invited, and 14 had consented, but we are unable to comment on how the educational or socio-economic status of those declining to participate differed compared with those who consented. As long-term conditions affect all social groups, non-participants' views may have differed from those reported here; they may have cultural reasons for not participating, being deterred by the inclusion criteria that both parents would participate or reluctant to discuss caregiving (Swallow 2008). We should consider ways to make research participation appealing to fathers and mothers from all social groups to elicit the broadest possible range of views. As is consistent with quali- 
tative research, we did not use random sampling therefore cannot comment on the frequency of views reported here. Nevertheless, we used recognized techniques to ensure the study's rigour. Like others (Costigan \& Cox 2001), we found many parents in our sample were graduates so may have been more likely to participate in research, although we found no noticeable difference between their accounts and those of nongraduate parents.

Future longitudinal research involving a larger sample and using quantitative and qualitative approaches, representing a wider range of long-term conditions, ethnic and socioeducational groups could examine, over time, the findings presented here in more detail. This may uncover changing patterns of caregiving or illustrate transfer of responsibilities between mothers and fathers (and children) at key times of transition, thereby informing professionals strategies to support fathers' and mothers' caregiving contributions.

\section{Conclusion}

Developing skills for home-based caregiving of long-term conditions was a challenging and uncertain process which parents negotiated with each other in order to provide mutual practical and emotional support. Both parents often participated in caregiving, developing skills in information processing, sharing/negotiating caregiving, restraining children, adapting to treatment regimens and communicating; they preferred to receive information directly from professionals rather than receiving it 'second hand' from each other. Fathers often assumed the 'protector' role, worrying about their child's long-term well-being; mothers worried more about current clinical issues and maintaining relationships with professionals.

\section{Key messages}

- Fathers and mothers participate in caregiving, developing skills in information processing, sharing/negotiating caregiving, restraining children, adapting to treatment regimens and communicating; they preferred to receive information directly from professionals rather than receiving it 'second hand' from each other.

- Developing skills is often a challenging and uncertain process, which parents negotiate with each other in order to provide mutual practical and emotional support.
- Fathers often assume the 'protector' role, worrying about their child's long-term well-being; mothers worry more about current clinical issues and maintaining relationships with professionals.

- Further longitudinal research involving a larger sample, representing a wider range of long-term conditions, ethnic and socio-educational groups may uncover changing patterns of caregiving by fathers as well as mothers, thereby informing professionals strategies to support both parents' caregiving contributions.

\section{Acknowledgements}

Grateful thanks to the parents who participated, the British Renal Society (Grant 06-017) and the Children's Kidney Unit Fund, Newcastle upon Tyne, UK who supported the project and Mr Roger Olley from Children North East who was adviser to the project.

\section{References}

Alderson, P. (2005) Designing ethical research with children. In: Ethical Research with Children (ed. A. Farrell), pp. 27-37. Open University Press, Maidenhead, UK.

Aldridge, M. (2008) How do families adjust to having a child with chronic kidney failure? A systematic review. Nephrology Nursing Journal, 35, 157-162.

Allmark, P. (2002) The ethics of research with children. Nurse Researcher, 10, 7-19.

Arockiasamy, V., Holsti, L. \& Albersheim, S. (2008) Fathers' experiences in the neonatal intensive care unit: a search for control. Pediatrics, 121, 215-222.

Barakat, L., Alderfer, M. \& Kazak, A. (2006) Posttraumatic growth in adolescent survivors of cancer and their mothers and fathers. Journal of Pediatric Psychology, 31, 413-419.

Burgess, A. (2005) Fathers and public services. In: Daddy Dearest? Active Fatherhood and Public Policy (ed. K. Stanley), pp. 57-74. Institute for Public Policy Research, London, UK.

Census-Bureau (2004) Current Population Reports. Census-Bureau, Washington, DC, USA.

Chesla, C. (1995) Hermeneutic phenomenology: an approach to understanding families. Journal of Family Nursing, 1, 63-75.

Chesler, M. A. \& Parry, C. (2001) Gender roles and/or styles in crisis: an integrative analysis of the experiences of fathers of children with cancer. Qualitative Health Research, 11, 363-384.

Clark, S. \& Miles, M. (1999) Conflicting responses: the experiences of fathers of infants diagnosed with severe congenital heart disease. Journal for Specialists in Pediatric Nursing, 4, 7-14.

Costigan, C. \& Cox, M. (2001) Fathers' participation in family research: is there a self-selection bias? Journal of Family Psychology, $15,706-720$. 
Department of Health (DoH) (2004) The National Service Framework for Renal Services (Part One) Dialysis and Transplantation.

Department of Health, London, UK.

Department of Health (DoH) (2006) The National Service Framework for Renal Services: Working for Children and Young People. Department of Health, London, UK.

Eiser, C. \& Havermans, T. (1992) Mothers and fathers coping with chronic childhood-disease. Psychology \& Health, 7, 249-257.

Eiser, C., Havermans, T., Pancer, M. \& Eiser, J. R. (1992) Adjustment to chronic disease in relation to age and gender: mothers' and fathers' reports of their children's behavior. Journal of Pediatric Psychology, 17, 261-275.

Gallo, A. M. \& Knafl, K. A. (1998) Parents' reports of 'tricks of the trade' for managing childhood chronic illness. Journal of the Society of Pediatric Nurses, 3, 93-100.

Gavin, L. \& Wysocki, T. (2006) Associations of paternal involvement in disease management with maternal and family outcomes in families with children with chronic illnesses. Journal of Pediatric Psychology, 31, 481-489.

Green, J. \& Thorogood, N. (2004) Qualitative Methods for Health Research. Sage, London, UK.

Hammersley, M. (1992) What's Wrong with Ethnography? . Routledge, London, UK.

Hill, K., Higgins, A., Dempster, M. \& McCarthy, A. (2009) Fathers' Views and understanding of their roles in families with a child with acute lymphoblastic leukaemia: an interpretative phenomenological analysis. Journal of Health Psychology, 14, 1268-1280.

Hovey, J. K. (2006) Differences in parenting needs of fathers of children with chronic conditions related to family income. Journal of Child Health Care, 10, 43-54.

Knafl, K. \& Zoeller, L. (2000) Childhood chronic illness: a comparison of mothers' and fathers' experiences. Journal of Family Nursing, 6, 287-302.

McBride, B. A., Brown, G. L., Bost, K. K., Shin, N., Vaughn, B. \& Korth, B. (2005) Paternal identity, maternal gatekeeping, and father involvement. Family Relations, 54, 360-372.

McGrath, P. \& Chesler, M. (2004) Fathers' perspectives on the treatment for pediatric hematology: extending the findings. Issues in Comprehensive Pediatric Nursing, 27, 39-61.

McIntosh, N., Bates, P. \& Brykczynska, G. (2000) Guidelines for the ethical conduct of medical research involving children. Royal College of Paediatrics, Child Health: Ethics Advisory Committee. Archives of Disease in Childhood, 82, 177-182.

Miller, D. \& MacDonald, D. (2006) Management of pediatric patients with chronic kidney disease. Nephrology Nursing Journal, 33, 128-134.

Neil-Urban, S. \& Jones, J. (2002) Father-to-father support: fathers of children with cancer share their experience. Journal of Pediatric Oncology Nursing, 19, 97-103.

Office of National Statistics (ONS) (2009) Social Trends. Office of National Statistics, London, UK.

Peck, B. \& Lillibridge, J. (2005) Normalization behaviours of rural fathers living with chronically-ill children: an Australian experience. Journal of Child Health Care, 9, 31-45.
Pelchat, D., Lefebvre, H. \& Perreault, M. (2003) Differences and similarities between mothers' and fathers' experiences of parenting a child with a disability. Journal of Child Health Care, 7, 231-247.

Pelchat, D., Lefebvre, H. \& Levert, M.-J. (2007) Gender differences and similarities in the experience of parenting a child with a health problem: current state of knowledge. Journal of Child Health Care, 11, 112-131.

Racher, F., Kaufert, J. \& Havens, B. (2000) Conjoint research interviews with frail, elderly couples: methodological implications. Journal of Family Nursing, 6, 367-378.

Ramchandani, P. \& McConachie, H. (2005) Mothers, fathers and their children's health. Child: Care, Health and Development, 31, $5-6$.

Ritchie, J. \& Lewis, J. (eds) (2003) Qualitative Research Practice: A Guide for Social Science Students and Researchers. Sage Publications, London, UK.

Ritchie, J. \& Spencer, L. (1994) Qualitative Data Analysis for Applied Policy Research. Analyzing Qualitative Data (eds A. Bryman \& R. G. Burgess), pp. 173-194. Routledge, London, UK.

Soliday, E., Kool, E. \& Lande, M. B. (2001) Family environment, child behavior, and medical indicators in children with kidney disease. Child Psychiatry and Human Development, 31, 279-295.

Sullivan-Bolyai, S., Rosenberg, R. \& Bayard, M. (2006) Fathers' reflections on parenting young children with type 1 diabetes. MCN. The American Journal of Maternal Child Nursing, 31, 24-31.

Swallow, V. (2008) An exploration of mothers' and fathers' views of their identities in chronic-kidney-disease management: parents as students? Journal of Clinical Nursing, 17, 3177-3186.

Swallow, V., Newton, J. \& Van Lottum, C. (2003) How to manage and display qualitative data using 'Framework' and Microsoft (R) Excel. Journal of Clinical Nursing, 12, 610-612.

Swallow, V., Lambert, H., Clarke, C., Campbell, S. \& Jacoby, A. (2008) Childhood chronic-kidney-disease: a longitudinal-qualitative study of families learning to share management early in the trajectory. Patient Education and Counseling, 73, 354-362.

Swallow, V., Macfadyen, A., Lambert, H. \& Santacroce, S. (2009) Fathers and mothers of children with long term kidney conditions: a qualitative study of their contributions to their child's healthcare (nephrology and clinical genetics joint session). Archives of Disease in Childhood, 94 (Suppl. 1), A34-A37.

Swallow, V., Macfadyen, A., Santacroce, S. \& Lambert, H. (2011) Fathers' contributions to management of their children's long-term medical conditions: a narrative review of the literature. Health Expectations (in press).

Swallow, V. M. \& Jacoby, A. (2001) Mothers' coping in chronic childhood illness: the effect of presymptomatic diagnosis of vesicoureteric reflux. Journal of Advanced Nursing, 33, 69-78.

Thomas, R. (1987) Methodological issues and problems in family health care research. Journal of Marriage and the Family, 49, $65-70$.

United Nations (UN) (1989) United Nations Convention on the Rights of the Child. United Nations, New York, NY, USA. 